\title{
Interações iniciais entre pais, mães e bebês de 0 a 3 anos: Revisão de literatura
}

Claudia Lúcia Menegatti. Pontifícia Universidade Católica do Paraná. Mara Albonei Dudeque Pianovski. Universidade Federal do Paraná. Suzane Schmidlin Löhr. Universidade Federal do Paraná.

\section{Resumo}

O estudo das interações iniciais dos bebês com seus cuidadores pode ser feito a partir da análise da responsividade parental. Com o objetivo de revisar os aspectos metodológicos e as tendências de análise das interações iniciais em estudos produzidos no Brasil, foram selecionados e analisados 110 artigos em bases eletrônicas de dados (Lilacs, SciELO, IndexPsi-Periódicos e Periódicos Capes). Os resultados apontaram para a predominância de estudos observacionais envolvendo as interações diádicas mãe-bebê. A coleta dos dados mais frequente foi por meio de combinação de instrumentos diretos e indiretos, e a análise se distribuiu de forma equivalente entre métodos qualitativos e quantitativos. Encontraram-se $55 \%$ de estudos sobre processos de desenvolvimento típico das interações e $45 \%$ de estudos com populações com risco biológico e social, abrindo questões para estudos futuros. Finalmente, sucedeu-se a análise dos fenômenos observados, levantando a discussão sobre os métodos de pesquisa na ciência psicológica.

Palavras-chave: relações pais-filhos; desenvolvimento infantil; pesquisa em psicologia.

\begin{abstract}
Early interactions between fathers, mothers and babies from 0 to 3 years old: Review of the literature. The study of early interactions between babies and their caretakers can be performed from the analysis of parental responsiveness. Aiming to review the methodological aspects and trends in the analyses of early interactions from studies performed in Brazil, 110 articles were chosen from electronic databases (Lilacs, SciELO, IndexPsi-Periódicos and Periódicos Capes) and then they were fully analyzed. The results revealed a predominance of observational studies involving dyadic mother-baby interactions. The most frequently form of data collection combined direct and indirect methods; and the analysis was equivalently distributed between qualitative and quantitative methods. Of the studies found, $55 \%$ of the studies focused on the developmental processes that are typical of interactions, and $45 \%$ of the studies focused on processes among populations at biological and social risk, which brought up relevant questions for future studies. Finally, the analysis of interactions raises discussion of the research methods in the psychological science.
\end{abstract}

Keywords: parent-chil relations; child development; psychology research.

\section{Resumen}

Interacciones iniciales entre padres, madres y bebés de 0 a 3 años: Revisión de literatura. El estudio de las interacciones iniciales de los bebés con sus cuidadores puede hacerse desde el análisis de la responsividad parental. Para revisar los aspectos metodológicos y tendencias de análisis de las interacciones iniciales en los estudios producidos en Brasil, se seleccionaron y analizaron 110 artículos en bases de datos electrónicas (Lilacs, SciELO, IndexPsi-Periódicos y Periódicos Capes). Los resultados señalaron el predominio de los estudios observacionales que implican interacciones diádicas madre-niño. La recolección de datos más frecuente fue a través de una combinación de instrumentos directos e indirectos, y el análisis se distribuyó de forma equivalente entre métodos cualitativos y cuantitativos. Se han encontrado $55 \%$ de estudios sobre los procesos de desarrollo típico de las interacciones y $45 \%$ de estudios con poblaciones con riesgo biológico y social, abriendo preguntas para futuros estudios. Por último, se hizo el análisis de los fenómenos observados, levantando la discusión sobre los métodos de investigación en la ciencia psicológica.

Palabras clave: relaciones padres-hijo; desarrollo infantil; investigación en psicología. 
Embora a revisão de literatura proposta neste artigo seja de trabalhos brasileiros sobre a temática da relação pais e filhos, o estudo das interações iniciais entre o bebê e seu cuidador foi mais profundamente considerado a partir das publicações do psiquiatra e psicanalista inglês John Bowlby na segunda metade do século XX (Bowlby,1969/1990; 1973/1998a; 1973/1998b). O trabalho de Bowlby, fundamentado em observação natural do comportamento da criança, consolidou a Teoria do Apego (Bowlby, 1969/1990), com posterior colaboração da norte-americana Mary Ainsworth (Ainsworth, Blehar, Waters, \& Wall, 1978). Essa formulação ofereceu parâmetros para observação das respostas afetivas entre mães e bebês, ampliando pesquisas sobre o desenvolvimento afetivo, cognitivo e social da criança (Eshel, Daelmans, Mello, \& Martines, 2006; Tamis-LeMonda, Bornstein, \& Baumwell, 2001; Tamis-LeMonda, Kuchirko, \& Song, 2014). Estudar as relações entre adultos e bebês, desde momentos muito precoces, possibilita compreender o impacto dessas interações no desenvolvimento global da criança, dado reconhecido em pesquisas clássicas internacionais (Ainsworth, 1989; Keller, 2014; Waters \& Cummings, 2000) e nacionais (Alvarenga \& Piccinini, 2007; Ribas \& Seidl de Moura, 2007). A partir desse contexto histórico-científico, foram revisados, no presente estudo, os marcos conceituais: apego, responsividade materna e bidirecionalidade das interações, enfatizando escolhas metodológicas de pesquisas nacionais e internacionais relevantes da área, o que fundamentou a compreensão das pesquisas produzidas no Brasil entre os anos de 1998 e 2015.

Conceitualmente, apego se refere a uma classe de comportamentos sociais que o bebê emite quando em interação, principalmente, com a mãe ou seu substituto. Resulta de uma propensão inata para o contato físico com outro ser humano, estabelecendo um vínculo intenso e profundo entre eles. Quando o bebê necessitar de aconchego, alimentação, cuidado ou proteção em situações estranhas e/ou estressantes, procurará estar próximo desse adulto (Bowlby, 1969/1990). O sistema comportamental do apego evoluiu por meio da seleção natural, aumentando as chances de proteção e sobrevivência da criança (Ainsworth, 1989).

O apego pode ser observado a partir da emissão de comportamentos de quem se sente 'apegado', com função de conservar ou obter a proximidade de um adulto diferenciado (Bowlby, 1973/1998b), geralmente expressados, no caso do bebê, por meio de respostas de choro e agarramento aos adultos, particularmente em situações estressantes. No entanto, como Keller (2014) assinalou criticamente, o próprio Bowlby frisava que o apego não se referia a respostas estereotipadas, mas a um desempenho particular da pessoa em seu ambiente.

Ainsworth (1989) destacou que essa classe de comportamentos infantis é dirigida ao principal cuidador ou a outras pessoas significativas muito selecionadas na relação com o bebê. Meehan \& Hawks (2014) fizeram menção a essa situação resgatando os estudos de Bowlby, Ainsworth e seus seguidores, nos quais estes claramente reconheceram que um bebê pode ter múltiplas figuras de apego, e que, mesmo assim, a maioria das pesquisas persiste analisando prioritariamente as relações mãe-bebê.

Os estudos de Bowlby, na Inglaterra, foram influenciados pela etologia e metodologicamente estruturados pela observação dos comportamentos infantis em ambiente natural (Van Der Horst, Van Der Veer, \& Van IJzendoorn, 2007). Já Ainsworth et al. (1978), no contexto norte-americano, propuseram método de investigação das reações dos bebês por meio de observação experimental, colocando-os em condições-teste para avaliar suas reações diante de afastamento e reaproximação de sua mãe ou substituto(a). Esse experimento, chamado situação estranha, consiste em um protocolo de episódios de aproximação/afastamento da figura materna e de um adulto estranho ao bebê, registrando as reações do bebê. Os resultados desses experimentos fundamentaram a classificação de tipos de apego seguro e inseguro, e também uma classe especial de comportamentos maternos denominada de sensitividade materna.

Sensitividade materna foi a primeira grande dimensão de observação de comportamentos maternos estabelecida por Ainsworth et al. (1978), e se referia às respostas maternas sensíveis aos sinais comunicacionais do bebê, que são a base para o desenvolvimento do apego seguro ou inseguro (Keller, 2014). A sensitividade materna é um construto relativo às dimensões de afetividade, calor e sensibilidade materna, assim como de prontidão para atender aos sinais do bebê contingentemente (Ribas \& Seidl de Moura, 2004). Pesquisadores brasileiros, como Alvarenga e Piccinini (2007), defendem que a sensibilidade materna se refere "à atenção e percepção consistentes, interpretação acurada e resposta contingente e apropriada da mãe aos sinais da criança" (p. 106).

Desse conceito desdobraram-se outros, como: sensitividade, sensibilidade (Silva, Le Pendu, Pontes, $\&$ Dubois, 2002), responsividade materna (Bornstein, 
Tamis-LeMonda, Hahn \& Haynes, 2008, Tamis-LeMonda et al., 2014) e responsividade sensível (Van Den Boom, 1994). São conceitos que guardam relações entre si, quando abordados por diferentes autores (Bornstein \& Manian, 2013; Piccinini et al., 2001). Dentre os termos resultantes de sensitividade materna, o mais consistentemente utilizado é responsividade materna, tanto na literatura internacional (Bornstein et al., 2008; Eshel et al.,2006; Tamis-LeMonda et al.,2014) quanto na nacional (Ribas \& Seidl de Moura, 2004).

As definições de responsividade têm pontos em comum. Tamis-LeMonda et al. (2014) a definiram como prontidão e contingência da resposta aos comportamentos exploratórios e comunicativos do bebê, na qual a prontidão se refere à resposta materna em curto intervalo de tempo (crítico para que o bebê faça a ligação entre seu comportamento e a resposta do cuidador) e a contingência se define pela consonância à ação iniciada pelo bebê. É um processo contíguo, contingente e com aspectos didáticos, que revela a codependência entre o comportamento do bebê e a resposta parental (Bornstein et al., 2008). Ribas e Seidl de Moura (2004), pesquisadores brasileiros, apontaram que responsividade e sensibilidade têm definições próximas, pois ambas tratam tanto do tempo (contiguidade e contingência da resposta materna), quanto da qualidade da resposta (adequação e afetividade).

A antropóloga norte-americana Hrdy (2001), ressaltou enfaticamente que o grande protagonista da história das relações iniciais é o próprio bebê, ativo e também construtor da relação com seu cuidador, seja a mãe ou outra pessoa. Considerando-se isso, são mais frequentes na literatura estudos sobre as interações quando o cuidador é a mãe do bebê, sendo menos pesquisadas as relações com outros cuidadores. Estudos publicados no Brasil justificam esse fato, e alegam que a mãe é um elemento crítico desse sistema, responsável pela apresentação do ambiente ao bebê, seguindo seus comportamentos e habilidades, e conduzindo as interações (Lordelo, Fonseca, \& Araújo, 2000). Entre a mãe e o bebê se estabelece um padrão de reciprocidade e sincronia, no qual cada um participa provendo a interação de estímulos e experiências, com regularidade e intensidade características de cada díade (Alfaya \& Schermann, 2005). Com o propósito de analisar pontoa-ponto essas interações, pesquisas brasileiras indicam comportamentos maternos e infantis que participam dessa interação, incluindo: contatos visuais, respostas de contato corporal, fala, beijo, sorriso, expressões de afeto e cuidados maternos, bem como o choro, sorriso e vocalização dos bebês (Piccinini, Alvarenga, \& Frizzo, 2007; Seidl de Moura \& Ribas, 2007). Na revisão de Zamberlan (2002), características do bebê como temperamento, variações nos estados de alerta, bem como os fatores de idade e desenvolvimento influenciam na qualidade das interações mãe-bebê. Já sobre as características maternas, mais estáveis do que as infantis, a autora destaca que influenciam na qualidade das interações as classes de comportamento de cuidados e as relativas à responsividade. Por isso, considera-se que a "responsividade não é um construto único, mas um domínio de variáveis relacionadas" (Alvarenga \& Piccinini, 2007, p. 106), nas quais estão incluídos os comportamentos do cuidador e do bebê, a sintonia entre eles, as direções da estimulação que a mãe provê, e as variáveis contextuais - ambiente próximo e ambiente sociocultural (Seidl de Moura et al., 2004).

Faz-se necessário lembrar que a responsividade é característica comum aos pais ao redor do mundo, na qual a variabilidade cultural implicará na variabilidade dos padrões de comportamento parental. Tais padrões podem ser de exploração guiada (extradiádicos) ou de conexão com outros (diádicos) (Tamis-LeMonda et al., 2014). Assim, a pesquisa sobre interações iniciais, à medida que analisa comportamentos do adulto, da criança e a dinâmica entre eles, requer desenhos metodológicos que contemplem a compreensão dos eventos passo-a-passo, como também a qualidade e variabilidade dos fenômenos envolvidos.

Ribas et al. (2003) e Seidl de Moura e Ribas (2007) apresentaram revisões nas quais encontraram que a metodologia mais empregada nos estudos sobre interação mãe-bebê são as observações naturalísticas ou em laboratório, compreendendo-as como metodologias adequadas aos propósitos de pesquisa nessa área. Esse dado vai de encontro às afirmações de Waters e Cummings (2000), pesquisadores norte-americanos que revisaram o legado das proposições de Bowlby e de Ainsworth e apresentaram os desafios para que as pesquisas da área sigam avançando com coerência. Segundo esses autores, as observações naturalísticas dos comportamentos de base segura são o padrão-ouro para avaliação e validação de medidas.

Além disso, as decisões metodológicas estão também relacionadas aos diferentes enfoques teóricos que se ocupam do estudo do desenvolvimento da criança (Zamberlan, 2002). Assim, observa-se um campo de pesquisa amplo e com formas de abordar, inferir, 
observar e analisar a relação mãe-bebê muito diferentes entre si, discussão presente na literatura internacional (Keller, 2014; Thompson, 2000) e nacional (Piccinini et al., 2001; Ribas, Seild de Moura, \& Ribas Jr, 2003). A literatura indica que, mesmo em diferentes perspectivas teóricas, a interação entre pais e/ou mães e seus bebês pode ser mais amplamente compreendida ao se considerar a reciprocidade e a interdependência entre os fenômenos comportamentais das partes envolvidas, compreendendo-os como bidirecionais. O estudo de Cohn e Tronick (1988), na realidade norte-americana, ilustra a emergência do debate científico sobre a bidirecionalidade de trocas interativas mãe-bebê. $O$ conceito da bidirecionalidade é destacado em estudos brasileiros (Piccinini et al., 2001; Seidl de Moura et al., 2004), resultando em pesquisas por meio de registros das interações em filmagens e a construção de protocolos com intervalos de tempo, a fim de verificar contiguidade e contingência das respostas interativas (Alfaya \& Schermann, 2005; Piccinini, Alvarenga, \& Frizzo, 2007). Consequentemente, as características maternas e infantis podem ser analisadas com diferentes caminhos metodológicos: por meio de microanálises e macroanálises, filmadas e codificadas, observadas em ambiente natural ou mesmo relatadas pelos seus responsáveis.

Ilustrando a utilização de filmagens e microanálises sobre diferentes índices de responsividade do adulto ao bebê, encontram-se exemplos de estudos internacionais (Beebe et al., 2010; Bornstein \& Manian, 2013) e nacionais (Piccinini Marin et al., 2007; Silva et al., 2002). Tais índices conduzem a análises sobre responsividade excessiva (super contingência, relacionada à intrusividade) ou deficiente (baixa contingência, relacionada à pouca afetividade) (Bornstein et al., 2008; Bornstein \& Manian, 2013; Troutman, 2015). Um exemplo é o estudo que foi conduzido por Bornstein \& Manian (2013), na realidade norte-americana, com 335 bebês de 4,7 a 6,5 meses e suas mães, para avaliar relações entre responsividade materna - por meio de microanálises das interações - e sensibilidade materna - por meio de classificação em uma macroanálise feita por observação das interações filmadas. Seus resultados foram que responsividade e sensibilidade andaram juntas até um ponto, quando, a partir dele, quanto mais responsividade, menor foi a sensibilidade.

No entanto, estudar o campo das interações significa observar como essas relações se estabelecem, desde uma análise ponto-a-ponto até uma análise dos significados mais amplos e contextos onde as interações ocorrem (Fuertes, Faria, Soares, \& Oliveira-Costa, 2010), o que constitui o espaço das macroanálises e das pesquisas de cunho qualitativo.

Schermann (2007) revisou estudos nacionais e internacionais sobre a proposição de métodos qualitativos e quantitativos na avaliação da interação mãe-bebê, de acordo com os propósitos específicos de cada pesquisa. Segundo a autora, a avaliação das interações pode ser realizada por métodos diretos e indiretos. Os métodos diretos coletam dados durante a própria interação, registrando-os de acordo com protocolos específicos. Já os métodos indiretos coletam informações por meio de entrevistas, inventários e questionários, possibilitando levantar também aspectos subjetivos presentes nos relatos.

Do ponto de vista de Martins Filho e Barbosa (2010), a pesquisa com crianças pequenas é um desafio metodológico emergente e abre questões sobre o uso de métodos qualitativos e quantitativos. Assim, recomendam seguir uma perspectiva interativa e não de submissão da criança ao adulto ou vice-versa, com reciprocidade e equilíbrio. Para esses autores, a metodologia qualitativa parece ser a mais apropriada para a compreensão da infância. No entanto, métodos quantitativos têm condições de prover informações generalizáveis e comparativas indispensáveis à compreensão do comportamento (Souza \& Gomes, 2003) e, como já visto, são muito utilizados nas observações das interações iniciais. Esse é um debate ainda vigente nas ciências sociais, inclusive o questionamento sobre a possibilidade de combinação de métodos qualitativos e quantitativos (Minayo, 2001).

Dada a relevância em descrever e elaborar práticas de atenção para bebês, mães, pais e cuidadores aloparentais, como recomenda o manual da World Health Organization (1997), o presente estudo pretendeu contribuir para o debate sobre os rumos na pesquisa nacional das interações iniciais. Pretendeu-se elucidar as seguintes questões: Que metodologias estão sendo utilizadas em pesquisa visando a compreensão desse fenômeno na realidade brasileira? Quais são as populações-alvo mais frequentemente estudadas? Quais são as temáticas das pesquisas sobre interações entre pais, mães e bebês de 0 a 3 anos?

\section{Método}

O presente estudo se caracteriza como uma revisão sistemática de literatura, conforme recomendam Sampaio e Mancini (2007). A revisão foi sistematizada a partir da busca de artigos disponibilizados eletronicamente com texto integral no Brasil até junho de 2015. 
Em função da variabilidade temporal em que cada periódico passou a disponibilizar seus trabalhos virtualmente, foram obtidos artigos desde 1998. As bases de dados utilizadas foram: Lilacs, SciELO e IndexPsi-Periódicos via BVS (Biblioteca Virtual da Saúde) e Periódicos Capes. A partir dos descritores da BVS para relacionamento pai - mãe-filho, foram definidos os termos de pesquisa: 'interação mãe-bebê'; 'interação pai-bebê'; 'relação mãe-bebê'; 'relação pai-bebê'; 'responsividade materna'; 'responsividade paterna'; 'sensibilidade materna'; 'sensibilidade paterna', por se referirem especificamente às variáveis de interesse do estudo. Os critérios de inclusão foram: artigos científicos de produção nacional e publicados com texto integral por meio eletrônico em periódicos nacionais; termos de pesquisa literalmente expressos no título ou resumo ou palavras-chave; artigos desenvolvidos sobre a relação parental com bebês de 0 a 3 anos de idade. Os critérios de exclusão foram: artigos não disponibilizados eletronicamente com texto integral; teses, dissertações, livros e capítulos de livros; artigos que não contivessem literalmente os termos de pesquisa no título, resumo ou palavras-chave; estudos desenvolvidos com crianças acima de 3 anos de idade, ou que se referiam a contextos escolares, ou que não se referiam a produções com dados nacionais.

Cada artigo foi analisado e os dados lançados em planilha eletrônica Excel para as comparações necessárias. As categorias estabelecidas para atingir os objetivos da pesquisa, após leitura e releitura integral dos artigos incluídos nesta revisão, foram: a) ano de publicação; b) natureza da pesquisa e método; c) natureza da coleta de dados; d) população-alvo das pesquisas; e) temáticas das pesquisas.

A categoria (b) considerou a natureza das pesquisas (teórica ou empírica, observacional ou experimental), e a escolha metodológica qualitativa ou quantitativa.

A categoria (c) natureza da coleta de dados foi dividida em quatro subcategorias: c1) Exclusivamente da literatura (para estudos de revisão de literatura narrativa ou sistemática); c2) Relato dos pais ou outros (consideraram-se relatos prestados pelos pais ou outros cuidadores, por meio de entrevistas, ou instrumentos padronizados de autorrelato/escalas psicológicas); e c3) Observação da interação (consideraram-se observações do comportamento da criança e/ou do adulto, diretas ou por meio de filmagens); c4) Relatos dos pais e observação da interação (relatos e observações como fontes das informações no mesmo estudo). Os locais onde os dados foram coletados foram analisados e agrupados em: residência dos participantes ou outros contextos de pesquisa.

A categoria (d) população-alvo da pesquisa foi dividida em três subcategorias: 1 ) estudo dirigido às mães e bebês (incluindo gestantes ou mães ou mãesbebês); d2) estudos dirigidos aos pais e mães (incluindo tríade pai-mãe-bebê ou pais e mães ou família); d3) estudos dirigidos aos pais e bebês (incluindo pais, paisbebês, pais 'grávidos').

A categoria (e) temáticas das pesquisas foi dividida em duas grandes subcategorias: e1) Conhecimento de processos e de desenvolvimento humano típicos (estudos sobre processos de desenvolvimento típico, podendo ser teóricos ou empíricos); e e2) Conhecimento de processos de desenvolvimento em populações especiais, minoritárias ou de risco (estudos sobre os processos de desenvolvimento das interações em situações de risco ou dirigido a populações que tenham risco presumido na literatura para dificuldades de desenvolvimento, problemas de saúde dos envolvidos ou em risco psicossocial. O risco presumido na literatura se refere a condições especiais maternas, paternas ou do bebê). Os dados obtidos foram descritivamente analisados.

\section{Resultados}

A revisão foi sistematizada considerando os artigos publicados e disponibilizados eletronicamente no Brasil até junho de 2015. Nas bases de dados consultadas foram encontrados 590 resumos a partir dos termos de pesquisa, sendo 445 na Lilacs, SciELO e IndexPsi-Periódicos via BVS, e 145 no Periódicos Capes. Depois de descartar os artigos repetidos, foram selecionados aqueles publicados integralmente por via eletrônica. A pesquisa foi realizada na segunda quinzena do mês de junho 2015. Os resumos foram lidos e então selecionados 140 artigos que continham os termos de pesquisa no título ou resumo ou nas palavras-chave. Após sua leitura integral, foram excluídos 30 artigos que não atendiam aos critérios de inclusão previamente definidos. Entre os 110 trabalhos que compuseram este estudo, 52 foram resultantes dos termos de pesquisa interação mãe-bebê e/ou interação pai-bebê, 43 decorrentes dos termos de pesquisa relação mãe-bebê e/ou relação pai-bebê; 12 com termos de pesquisa responsividade materna e/ou responsividade paterna, e três com termos de pesquisa sensibilidade materna e/ou sensibilidade paterna.

As publicações encontradas foram agrupadas de seis em seis anos. Entre os anos de 1998 e 2003 concentraram-se $13 \%$ dos estudos $(n=14)$, entre 2004 
e 2009 situaram-se 48\% ( $n=53$ ), e, finalmente, no intervalo de 2010 a 2015 concentraram-se $39 \%(n=43)$ dos trabalhos analisados nesta pesquisa.

$\mathrm{Na}$ amostra total estudada $(n=110)$, os trabalhos se dividiram entre estudos empíricos, (68\%; $n=75)$, e estudos teóricos (32\%; $n=35$ ). Os estudos empíricos foram classificados quanto à sua natureza, sendo constatado que $92 \%(n=69)$ eram observacionais e somente $8 \%$ experimentais $(n=6)$. No total de estudos observacionais empíricos, foram incluídos os estudos descritivos (longitudinais ou transversais), estudos descritivos de comparação entre grupos contrastantes (longitudinais ou transversais) e estudos de caso. Nas pesquisas de natureza experimental $(n=6)$ observaramse delineamentos quase-experimentais e experimentais. Quanto ao método de análise dos dados, 47\% ( $n=$ 35) dos trabalhos empíricos se utilizaram de métodos quantitativos, $47 \%$ ( $n=47 \%$ ) de métodos qualitativos e $6 \%(n=5)$ de métodos quanti-qualitativos.

Ao analisar a natureza do método para a coleta de dados, verificou-se que foi mais frequente a utilização de relatos dos participantes (entrevistas ou medidas por meio de relatos verbais como questionários, escalas e testes psicológicos) associados a métodos de observação de comportamento dos mesmos (observações ao vivo ou por meio de filmagens dos participantes, as quais foram depois categorizadas e/ou analisadas), representando $37 \%(n=41)$ da amostra total $(n=110)$. Estudos que apoiaram suas conclusões somente a partir dos relatos corresponderam a $21 \%(n=23)$ da amostra total. Os trabalhos em que os dados foram coletados somente por meio de observações de comportamento totalizaram $10 \%$ ( $n=11$ ), e estudos exclusivamente teóricos, envolvendo as diferentes formas de revisão de literatura, corresponderam a $32 \%(n=35)$ do total de artigos (Figura 1).

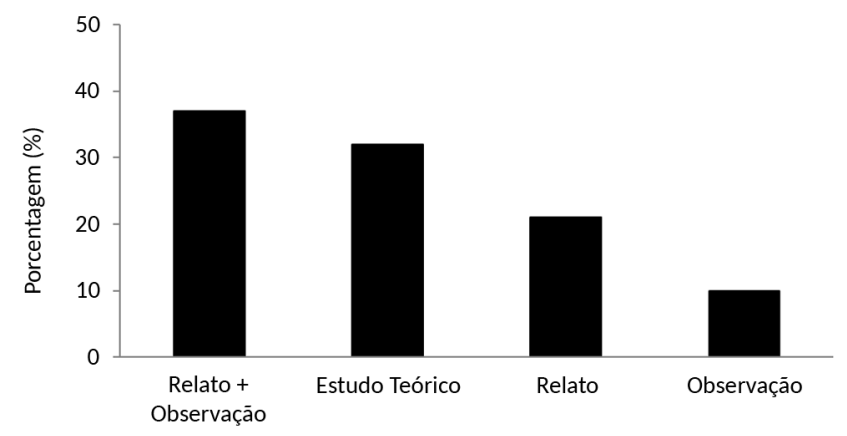

Figura 1. Percentuais Gerais da Natureza da Coleta de Dados nas 110 Pesquisas.
Os locais da coleta de dados dos trabalhos permitiram dividi-los em dois agrupamentos: residência dos participantes e outros contextos de pesquisa. Entre os 75 estudos empíricos observou-se que os autores realizaram a coleta de dados de suas pesquisas nas residências dos participantes em 53\% $(n=40)$ dos trabalhos. Essa escolha foi justificada por possibilitar a observação do comportamento no ambiente natural das crianças e pais, além de facilitar a coleta (possível aumento da adesão dos participantes aos estudos). Nos outros $47 \%(n=35)$, os autores realizaram a coleta de dados nos hospitais, ambulatórios ou nas universidades às quais os estudos estavam vinculados.

Quanto às populações-alvo, observou-se que os estudos dirigidos às mães e bebês representaram a grande maioria dos trabalhos, com $79 \%(n=85)$ da amostra total. Já os estudos dirigidos aos pais e mães (incluindo tríade pai-mãe-bebê, ou pais e mães, ou família) foram $16 \%(n=17)$ e, finalmente, os estudos dirigidos a pais e bebês representaram $5 \%(n=6)$ da amostra total.

Analisando as temáticas dos trabalhos, encontrou-se leve predomínio de estudos voltados à produção de conhecimento de processos e de desenvolvimento humano típicos $(n=61)$, representando $55 \%$ da amostra. Nesses estudos, os objetivos se dirigiram a descrever o desenvolvimento típico das interações entre cuidadores e seus bebês, sem a interferência de variáveis de risco maternas, paternas, infantis ou ambientais. Os estudos que se dirigiram ao conhecimento de processos de desenvolvimento em populações de risco, especiais ou minoritárias representaram $45 \%(n=49)$ da amostra total, identificadas na Tabela 1.

\section{Discussão}

Revisar uma temática na literatura de forma sistematizada auxilia a observação do estado da arte de um assunto e as lacunas para futuras pesquisas (Sampaio \& Mancini, 2007). O objetivo deste estudo foi revisar os aspectos metodológicos e os temas investigados nos estudos produzidos no Brasil sobre interações entre pais, mães e bebês de 0 a 3 anos de idade. Após a análise dos artigos incluídos, os principais resultados foram que o estudo das interações iniciais esteve, no Brasil, predominantemente voltado para as interações diádicas mãe-bebê, e adotou, em geral, metodologias observacionais.

Os tipos de estudos representados nesta análise foram mais frequentemente empíricos (68\%) e, de forma equilibrada, apresentaram análises qualitativas ou 
Tabela 1. Distribuição dos Estudos Dirigidos a Populações de Risco, Especiais ou Minoritárias.

\begin{tabular}{lcc}
\hline Populações de risco & Estudos (n) & Percentual (\%) \\
\hline Prematuros & 11 & $22 \%$ \\
Mães que apresentaram depressão pós-parto e materna & 11 & $22 \%$ \\
Bebês com necessidades especiais & 8 & $16 \%$ \\
Bebês com problemas de saúde relativos a cuidados (desnutrição, sintomas psicofuncionais, maus tratos) & 5 & $10 \%$ \\
Mães soropositivas para HIV & 5 & 4 \\
Mães apresentando outros problemas psiquiátricos (ansiedade, psicose) & 4 & $8 \%$ \\
Mães e/ou pais adolescentes & 4 & $8 \%$ \\
Mulheres com gestação após morte perinatal & 1 & $2 \%$ \\
Total & 49 & $100 \%$ \\
\hline
\end{tabular}

quantitativas. Sobre as medidas para coleta de dados, a combinação entre medidas diretas e indiretas foram mais frequentes. O local de coleta de dados teve frequência equilibrada entre os locais-sede das pesquisas realizadas e as residências dos participantes. As pesquisas para a compreensão de processos típicos de desenvolvimento foram as mais frequentes, e, entre os estudos de processos de desenvolvimento das interações em situações atípicas, encontraram-se temas como as interações iniciais nas condições de prematuridade, depressão pós-parto e bebês com necessidades especiais, principalmente.

A escolha metodológica de uma pesquisa não deve ser feita deliberadamente, mas pela observação da natureza do problema de estudo, compreendendo suas variáveis e a relevância dos resultados pretendidos (Weber, Botomé, \& Rebelatto, 1996). Nesta revisão, a grande maioria dos estudos analisados foi de natureza observacional, o que pode se relacionar à complexidade dos fenômenos psicológicos, custos e possíveis aspectos éticos envolvidos nas investigações experimentais (Tourinho, 2003). Nesta mesma perspectiva, mesmo mais frequentes na presente revisão, os estudos empíricos dividiram espaço com os estudos teóricos, reforçando a hipótese dos custos temporais e financeiros como complicadores das pesquisas empíricas, e, especificamente, das experimentais. Esta baixa frequência de experimentos recorda o alerta de Carolina Bori, relatado por Botomé (2007), sobre a responsabilidade coletiva dos pesquisadores em Psicologia no Brasil em propor trabalhos que estejam a serviço das necessidades da população. Portanto, é necessário investigar se há, no meio acadêmico brasileiro, conhecimento das recomendações do manual da World Health Organization (1997) sobre a prioridade, para a saúde dos bebês, em desenvolver intervenções focadas nas interações iniciais nos países em desenvolvimento, o que demandaria maior número de estudos empíricos.
O equilíbrio entre a frequência de pesquisas qualitativas e quantitativas nos trabalhos analisados tem relação com a natureza dos fenômenos estudados, pois as interações iniciais podem ser observadas quantitativamente para o controle de variáveis e com categorias pré-fixadas, quanto por meio de observações qualitativas para a apreensão mais global dos fenômenos (Turato, 2005). São diferentes vias de análise sobre os fenômenos que ocorrem nas interações iniciais com os bebês, e que podem trazer compreensões complementares, pois, se métodos quantitativos expõem ocorrências mais frequentes e gerais, os qualitativos se apoiam em ocorrências específicas ou singulares, como estudar populações especiais ou de risco. Com os recursos tecnológicos atualmente disponíveis (filmagens e programas computacionais de observação), a escolha recai sobre que tipo de dado pretende ser produzido (Günther, 2006). Minayo (2001) defende a postura de que os métodos quantitativos e qualitativos diferem quanto à sua natureza, porém, isso não significa que são opostos excludentes, pois a realidade que abrangem está em dinâmica interação.

Há uma influência histórica importante dos estudos iniciais de apego para a escolha de métodos de coleta de dados sobre interações iniciais, pois aqueles estudos se utilizavam da observação naturalística ou em ambientes controlados (p.ex., situação estranha de Ainsworth et al., 1978). Quando os estudos analisados neste trabalho utilizaram observações diretas das interações, buscaram formas de avaliação da contiguidade e contingência das respostas parentais, tendendo a privilegiar as observações filmadas e os registros de evento por intervalos de tempo. Schermann (2007) enfatizou que a metodologia observacional direta (ao vivo ou por meio de filmagens) é o método de excelência para a compreensão das interações, mesmo sendo bastante comum o uso combinado de métodos diretos e indiretos de obtenção de dados, como se observou neste trabalho. 
A natureza da coleta dos dados reunindo medidas diretas e indiretas reflete a complexidade dos fenômenos interativos e as diferentes orientações teóricas. Estudos baseados na psicanálise, por exemplo, privilegiaram o discurso dos participantes, mesmo quando combinado a observações das interações. Outros estudos empregaram escalas de autorrelato para avaliar fenômenos como a depressão e ansiedade maternas. Sobre essa questão, em sua revisão sobre estudos de interações iniciais, Wendland (2001) discute que, mesmo havendo divergências e limitações, há pouca rivalidade entre os métodos em função da complexidade do fenômeno de estudo.

As coletas de dados aconteceram em locais distintos: nas residências dos participantes e nas sedes das pesquisas (universidades, hospitais ou ambulatórios). Destacando-se aqui a utilização das residências como cenário de trabalho, a postura metodológica que considera a observação naturalística como forma privilegiada de análise das interações iniciais torna-se mais evidente (Seidl de Moura \& Ribas, 2007), o que também se observa na literatura internacional (Eshel et al., 2006). Alguns autores (Brum \& Schermann, 2007; Murta, Rodrigues, Rosa, \& Paulo, 2012) indicam, inclusive, que as visitas domiciliares na avaliação e intervenção sobre as interações familiares estão associadas ao sucesso de tais intervenções.

Houve predomínio de trabalhos envolvendo exclusivamente as interações entre mães e bebês, perspectiva mais privilegiada nos estudos, pois o apego é um fenômeno diadicamente organizado (Keller, 2014). No entanto, a partir do nascimento, os cuidados com o bebê se ampliam de diferentes formas em diferentes culturas, confirmando que poderão ter múltiplas figuras de apego familiares ou não, e questionando a crença anterior sobre a monotropia nas relações iniciais (Thompson, 2000). A presente revisão encontrou poucos estudos envolvendo a tríade pai-mãe-bebê e a díade pai-bebê. Razões para a exclusão dos pais em grande parte dos estudos parece se relacionar às facilidades de acesso às mães, e também ainda a uma manutenção do papel do pai como provedor da família e a mãe como cuidadora. Atualmente, esses papéis estão em modificação, e, em vários segmentos da sociedade, pode-se observar pais atuando cada vez mais nos cuidados dos filhos (Murta et al., 2012), tendência de pesquisa já indicada por Wendland (2001). Vieira et al. (2014) e Jager e Dias (2014) indicaram a necessidade de pesquisa sobre as variáveis que contribuem para a aproximação e construção interacional pai-bebê, indo além do senso comum sobre a importância do envol- vimento paterno nos cuidados prestados. Discussões envolvendo as interações triádicas foram feitas na literatura internacional por Waters e Cummings (2000), os quais destacaram a necessidade de estudo sobre apego no contexto familiar como um todo, entendendo que a formação do apego é multiplamente influenciada, por exemplo, pelos conflitos conjugais e nascimento de irmãos. Em função das mudanças familiares e das pressões econômicas sobre homens e mulheres, faz-se necessário ampliar os estudos brasileiros sobre apego nos próximos anos, incluindo temas como a interação com filhos de casais homoafetivos e a aloparentalidade, discussões já presentes na literatura internacional (Golombok et al., 2014; Meehan \& Hawks, 2014).

Quanto às temáticas estudadas, mesmo que tenham sido mais frequentes os estudos com populações em desenvolvimento típico, foram também produzidos estudos envolvendo populações especiais ou de risco. Entre estas, as mais estudadas foram as mães com depressão pós-parto e seus bebês, as interações com prematuros e com bebês com necessidades especiais, dado também encontrado por Wendland (2001). Ribas e Seidl de Moura (2007) já alertavam que os estudos sobre responsividade parental precisavam ser ampliados em duas vias: o debate da universalidade do construto responsividade e a ampliação da origem das populações estudadas, pois a Psicologia ainda carrega o estigma de 'Psicologia da classe média'. Mesmo havendo representatividade de estudos com prematuros e sobre interações iniciais na situação da depressão pós-parto, outras situações de risco biológico e social têm sido menos estudadas na realidade brasileira. A este respeito, Keller (2014) alertou criticamente que a maioria dos estudos sobre interações iniciais são realizados com crianças de raça branca, vindas de famílias com educação formal e em sociedades industrializadas. Isso inviabiliza a aplicação integral dos dados obtidos a outros contextos, como pobreza, maternidade precoce, bebês com doenças crônicas e sociedades com outros costumes na criação de filhos. Essa é uma reflexão sobre o fazer da ciência psicológica. Tourinho (2003), ao analisar a produção de conhecimento em Psicologia, frisa que o campo da Psicologia é multifacetado, pois apresenta diferentes discursos e atende a demandas muito diversas entre si. Assim, a produção científica deve estar filosoficamente articulada e prover elementos para intervenção em fenômenos humanos complexos, em diferentes vias de análise, para atender a contextos cientificamente também desprivilegiados. 


\section{Considerações finais}

Com o objetivo de revisar os aspectos metodológicos de artigos nacionais sobre interações iniciais, este estudo contribuiu para refletir sobre o panorama das pesquisas da área. Como grande parte dos trabalhos se dirigiu às interações mãe-bebê, alerta-se para a ampliação das pesquisas, incluindo pais, irmãos e cuidadores aloparentais. Outra contribuição se refere ao menor número de estudos com populações com riscos biológicos e/ou sociais, sendo essas, portanto, indicações para futuras pesquisas. Finalmente, é necessário estimular pesquisas com delineamentos experimentais, que forneçam subsídios para a elaboração de ações que atendam às necessidades de populações brasileiras específicas.

Uma limitação deste estudo foi a decisão sobre a definição dos termos para levantamento de artigos. Expressões como 'díade mãe-filho', 'dialogia mãe-bebê' e 'vínculo' poderiam trazer dados complementares aos já obtidos, e não foram incluídas, pois não atendiam aos termos previamente definidos. Como ora pretendeu-se conhecer o panorama metodológico dos estudos sobre as interações iniciais, privilegiando o conhecimento produzido sobre as dimensões da responsividade e sensibilidade, os objetivos foram atingidos e se beneficiarão de comparações futuras incluindo as expressões supramencionadas.

\section{Referências}

Ainsworth, M. D. S. (1989). Attachments beyond infancy. American Psychologist, 44(4), 709-716. doi: 10.1037/0003-066X.44.4.709

Ainsworth, M. D. S., Blehar, M. C., Waters, E., \& Wall, S. (1978). Patterns of attachment: a psychological study of the strange situation. Hillsdale: Erlbaum.

Alfaya, C., \& Schermann, L. (2005). Sensibilidade e aleitamento materno em díades com recém-nascidos de risco. Estudos de Psicologia, 10(2), 279-285. doi:10.1590/S1413-294X2005000200015

Alvarenga, P., \& Piccinini, C. A. (2007). Preditores do desenvolvimento social na infância: potencial e limitações de um modelo conceitual. Interação em Psicologia, 11(1), 103-112. doi: 10.5380/psi.v11i1.7761

Beebe, B., Jaffe, J., Markese, S., Buck, K., Chen, H., Cohen, P., \& Feldstein, S. (2010). The origins of 12-month attachment: a microanalysis of 4-month mother-infant interaction. Attachment \& Human Development, 12(0), 3-141. doi:10.1080/14616730903338985

Bornstein, M. H., \& Manian, N. (2013). Maternal responsiveness and sensitivity reconsidered: some is more. Development and Psychopathology, 25(4), 957-971. doi:10.1017/S0954579413000308

Bornstein. M. H., Tamis-LeMonda, C. S., Hahn, C. S., \& Haynes, N. O. (2008). Maternal responsiveness to young children at three ages: longitudinal analysis of a multidimensional, modular, and specific parenting construct. Developmental Psychology, 44(3), 867-874. doi: 10.1037/0012-1649.44.3.867
Botomé, S. P. (2007). Onde falta melhorar a pesquisa em Psicologia no Brasil sob a ótica de Carolina Martuscelli Bori. Psicologia: Teoria e Pesquisa, 23(número especial), 29-40. doi: 10.1590/S010237722007000500006

Bowlby, J. (1990). Apego: a natureza do vínculo (2a ed, vol. 1). São Paulo: Martins Fontes (Obra original publicada em 1969)

Bowlby, J. (1998a). Separação: angústia e raiva (3ª ed, vol.2). São Paulo: Martins Fontes (Obra original publicada em 1973)

Bowlby, J. (1998b). Perda: tristeza e depressão (2a ed, vol. 3). São Paulo: Martins Fontes (Obra original publicada em 1973)

Brum, E. H. M., \& Schermann, L. (2007). Intervenção para promover a qualidade do vínculo mãe-bebê em situação de nascimento pré-termo. Revista Brasileira de Crescimento e Desenvolvimento Humano, 17(2), 12-23. Recuperado de http://www.revistas.usp.br/ jhgd/article/viewFile/19828/21900

Cohn, J. F., \& Tronick, E. Z. (1988). Mother-infant face-to-face interaction: influence is birectional and unrelated to periodic cycles in either partner's behavior. Developmental Psychology, 24(3), 386-392. doi: 10.1037/0012-1649.24.3.386

Eshel, N., Daelmans, B., Mello, M. C., \& Martines, J. (2006). Responsive parenting: interventions and outcomes. Bulletin of the World Health Organization, 84(12), 991-998. doi:10.1590/S004296862006001200016

Fuertes, M., Faria, A., Soares, H., \& Oliveira-Costa, A. (2010). Momentos de interação em que as emoções se apre(e)ndem: estudo exploratório sobre a prestação materna e infantil em jogo livre. Psicologia USP, 21(4), 833-857. doi: 10.1590/S0103-65642010000400010

Golombok, S.. Mellish, L.; Jennings, S., Casey, P., Tasker, F., \& Lamb, M. E. (2014). Adoptive Gay Father Families: parent-child relationships and children's psychological adjustment. Child Development, 85(2), 456-468. doi: 10.1111/cdev.12155

Günther, H. (2006). Pesquisa qualitativa versus pesquisa quantitativa: esta é a questão? Psicologia: Teoria e Pesquisa, 22(2), 201-210. doi: $10.1590 /$ S0102-37722006000200010

Hrdy, S. B. (2001). Mothers and others: from queen bees to elephant matriarchs, many animal mothers are assisted by others in rearing offspring. Natural History Magazine, 110(4), 50-63. Recuperado de https://archive.org/stream/naturalhistory11004unse\#page/50/ mode/2up

Jager, A. E., \& Dias, A. C. G. (2014). Paternidade adolescente e o envolvimento paterno na perspectiva bioecológica do desenvolvimento humano. Pensando Famílias, 18(1), 45-54. Recuperado de http:// pepsic.bvsalud.org/scielo.php?script=sci_arttext\&pid=S1679-494X2 014000100005\&lng=pt\&nrm=iso.

Keller, H. (2014). Introduction: understanding relationships - what we would need to know to conceptualize attachment as the cultural solution of a universal developmental task. In H. Otto \& H. Keller (Orgs.), Different faces of attachment - cultural variations on a universal human need. (pp. 1-21). Reino Unido: Cambridge University Press.

Lordelo, E. R., Fonseca, A. L., \& Araújo, M. L. V.B. (2000). Responsividade do ambiente de desenvolvimento: crenças e práticas como sistema cultural de criação de filhos. Psicologia: Reflexão e Crítica, 3(1), 7380. doi:10.1590/S0102-79722000000100009

Martins Filho, A. J., \& Barbosa, M. C. S. (2010). Metodologias de pesquisas com crianças. Reflexão e Ação, 18(2), 8-28. doi: 10.17058/ rea.v18i2.1496 
Meehan, C. L., \& Hawks, S. (2014). Maternal and allomaternal responsiveness: the significance of cooperative caregiving in attachment theory. In H. Otto \& H. Keller (Orgs.), Different faces of attachment - cultural variations on a universal human need. (pp.113-140). Reino Unido: Cambridge University Press.

Minayo, M. C. S. (Org.) (2001). Pesquisa Social - Teoria, método e criatividade (18 ed.) Petrópolis: Vozes.

Murta, S. G., Rodrigues, A. C., Rosa, I. O., \& Paulo, S. G. (2012). Avaliação de um programa psicoeducativo de transição para a parentalidade. Paideia, 22(53), 403-412. doi:10.1590/S0103-863X2012000300012

Piccinini, C. A., Alvarenga, P., \& Frizzo, G. B. (2007). Responsividade como foco de análise da interação mãe-bebê e pai-bebê. In C. A. Piccinini \& M.L. S. Moura (Orgs.), Observando a interação pais-bebê-criança (pp. 131-153). São Paulo: Casa do Psicólogo.

Piccinini, C. A., Marin, A. N., Alvarenga, P., Lopes, R. C. S., \& Tudge, J. R. (2007). Responsividade materna em famílias de mães solteiras e famílias nucleares no terceiro mês de vida da criança. Estudos de psicologia, 12(2), 109-117. doi: 10.1590/S1413-294X2007000200002

Piccinini, C. A., Seidl de Moura, M. L., Ribas, A. F. P., Bosa, C. A., Oliveira, E. A., Pinto, E. B., \& Chahon, V. L. (2001). Diferentes perspectivas na análise da interação pais-bebê/criança. Psicologia: Reflexão e Crítica, 14(3), 469-485. doi:10.1590/S0102-79722001000300004

Ribas, A. F.P., \& Seidl de Moura, M. L. (2004). Responsividade materna e teoria do apego: uma discussão crítica do papel de estudos transculturais. Psicologia: Reflexão e Crítica, 17(3), 315-322. doi:10.1590/ S0102-79722003000100014

Ribas, A. F. P., \& Seidl de Moura, M. L. (2007). Responsividade materna: aspectos biológicos e variações culturais. Psicologia: Reflexão e Crítica, 20(3), 368-375. doi: 10.1590/S0102-79722007000300004

Ribas, A. F.P., Seidl de Moura, M. L., \& Ribas Jr, R. C. (2003).Responsividade materna: levantamento bibliográfico e discussão conceitual. Psicologia: Reflexão e Crítica, 16(1), 137-145. doi: 10.1590/S010279722003000100014

Sampaio, R. F., \& Mancini, M. C. (2007). Estudos de revisão sistemática: um guia para síntese criteriosa da evidência científica. Revista Brasileira de Fisioterapia, 11(1), 83-89. doi:10.1590/S141335552007000100013

Schermann, L. (2007). Avaliação quantitativa e qualitativa da interação mãe-bebê. In C. A. Piccinini \& M. L. S. Moura (Orgs.), Observando a interação pais-bebê-criança (pp. 155-175). São Paulo: Casa do Psicólogo.

Seidl de Moura, M. L., \& Ribas, A. F.P. (2007). A pesquisa observacional e o estudo da interação mãe-bebê. In C. A. Piccinini \& M. L. Seidl de Moura (Orgs.), Observando a interação pais-bebê-criança (pp. 103-130). São Paulo: Casa do Psicólogo

Seidl de Moura, M. L., Ribas, A. F. P., Seabra, K. C., Pessoa, L. F., Ribas Jr., \& Nogueira, S. E. (2004). Interações iniciais mãe-bebê. Psicologia: Reflexão e Crítica, 17(3), 295-302. doi:10.1590/S010279722004000300002

Silva, S. S. C., Le Pendu, Y., Pontes, F. A. R., \& Dubois, M. (2002). Sensibilidade materna durante o banho. Psicologia: Teoria e Pesquisa, 18(3), 345-352. doi:10.1590/S0102-37722002000300015
Souza, M. L., \& Gomes, W. B. (2003). Evidência e interpretação em pesquisa: as relações entre qualidades e quantidades. Psicologia em Estudo, 8(2), 83-92. Recuperado de http://www.scielo.br/pdf/ pe/v8n2/v8n2a08.pdf

Tamis-LeMonda, C. S. T., Bornstein, M. H., \& Baumwell, L. (2001) Maternal responsiveness and children's achievement of language milestones. Child Development, 72(3), 748-767. doi: 10.1111/14678624.00313

Tamis-LeMonda, C. S. T., Kuchirko, Y. L., \& Song, L. (2014). Why is infant language learning facilitated by parental responsiveness? Current Directions in Psychological Science, 23(2), 121-126. doi: $10.1177 / 0963721414522813$

Thompson, R. A. (2000). The legacy of early attachments. Child Development, 71(1), 145-152. doi: 10.1111/1467-8624.00128

Tourinho, E. Z. (2003). A produção de conhecimento em Psicologia: a análise do comportamento. Psicologia, Ciência e Profissão, 23(2), 30-41. doi: 10.1590/S1414-98932003000200006

Troutman, B. (2015). Integrating behaviorism and attachment theory in parent coaching. lowa: Springer.

Turato, E. R. (2005). Métodos qualitativos e quantitativos na área da saúde: definições, diferenças e seus objetos de pesquisa. Revista de Saúde Pública, 39(3), 507-514. doi: 10.1590/S003489102005000300025

Van Den Boom, D. C. (1994). The influence of temperament and mothering on attachment and exploration: an experimental manipulation of sensitive responsiveness among lower-class mothers with irritable infants. Child Development, 65(5), 1457-77. doi: 10.1111/j.14678624.1994.tb00829.x

Van Der Horst, F. C. P., Van Der Veer, R., \& Van IJzendoorn, M. H. (2007) John Bowlby and ethology: an annotated interview with Robert Hinde. Attachment \& Human Development, 9(4), 321-335. doi: 10.1080/14616730601149809

Vieira, M. L., Bossardi, C. N., Gomes, L. B., Bolze, S. D. A., Crepaldi, M. A., \& Piccinini, C. A. (2014). Paternidade no Brasil: revisão sistemática de artigos empíricos. Arquivos Brasileiros de Psicologia, 66(2), 36-52. Recuperado de http://146.164.3.26/index.php/abp/ article/view/854/859

Waters, E., \& Cummings, E. M. (2000). A secure base from which to explore close relationships. Child Development, 71(1), 162-172. doi: 10.1111/1467-8624.00130

Weber, L. N. D., Botomé, S. P., \& Rebelatto, J. R. (1996). Psicologia: definições, perspectivas e desenvolvimento. Psicologia Argumento, 19, 9-28.

Wendland, J. A. (2001). A abordagem clínica das interações pais-bebê: perspectivas teóricas e metodológicas. Psicologia: Reflexão e Crítica, 14(1): 45-56. doi: 10.1590/S0102-79722001000100004

World Health Organization/World Health Organization Programme on Mental Health (1977). Improving mother/child interaction to promote better psychosocial development in children. Genebra: Autor

Zamberlan, M. A. T. (2002). Interação mãe-criança: enfoques teóricos e implicações decorrentes de estudos empíricos. Estudos de Psicologia, 7(2), 399-406. doi 10.1590/S1413-294X2002000200021 
C. L. Menegatti, M. A. D. Pianovski, S. S. Löhr

Claudia Lúcia Menegatti, Doutora em Saúde da Criança e do Adolescente pela Universidade Federal do Paraná (UFPR), é Professora da Pontifícia Universidade Católica do Paraná, (PUCPR).

Endereço para correspondência: Av. Água Verde, 1575, apto.

2101-1 - Água Verde - Curitiba PR - CEP: 80620-200. Telefones: (41) 30197422/99642-3538. E-mail: claudiamenegatti@gmail.com

Mara Albonei Dudeque Pianovski, Doutora em Saúde da Criança e do Adolescente pela Universidade Federal do Paraná (UFPR),

é Professora do Programa de Pós-Graduação em Saúde da

Criança e do Adolescente (UFPR) e Coordenadora do Centro de Projetos de Ensino e Pesquisa (CEPEP) da Liga Paranaense de Combate ao Câncer (LPCC). E-mail: mpianovski@lpcc.org.br

Suzane Schmidlin Löhr, Doutora em Psicologia Clinica pela Universidade de São Paulo (USP), Pós-doutorado em curso na Universidade de São Paulo (USP) e Universidade de Lisboa, é Professora Associada na Universidade Federal do Paraná (UFPR). E-mail: lohr@superig.com.br

Recebido em 29.Set.15

Revisado em 03.Nov.16

Aceito em 31.Dez.16 